\title{
Stability characteristics of conjugate natural convection boundary layers
}

\author{
N. Williamson ${ }^{1} \quad$ S. W. Armfield ${ }^{2}$
}

(Received 18 January 2011; revised 10 June 2011)

\begin{abstract}
Flow in a differentially heated two dimensional rectangular cavity, partitioned in the centre by an infinitely conducting vertical wall, has been examined with numerical simulations over Rayleigh numbers around $10^{10}$ at Prandtl number 7.5. The configuration is an idealised version of a flow which occurs commonly in engineering settings and is of fundamental importance. Heat is transferred between both sides of the cavity through the conducting wall with natural convection boundary layers forming on all vertical surfaces. We show for the first time that the flow becomes oscillatory above Rayleigh number $1.2 \times 10^{10}$ for cavity height to width ratio of two, and above Rayleigh number $1.4 \times 10^{10}$ for cavity height to width ratio of one. The results indicate that the instability is a convective boundary layer instability which becomes absolutely unstable as a result of the thermal coupling across the partition wall.
\end{abstract}

http://anziamj . austms.org.au/ojs/index.php/ANZIAMJ/article/view/3886 gives this article, (C) Austral. Mathematical Soc. 2011. Published August 16, 2011. ISSN 1446-8735. (Print two pages per sheet of paper.) Copies of this article must not be made otherwise available on the internet; instead link directly to this URL for this article. 


\section{Contents}

1 Introduction

C697

2 Numerical formulation

C699

3 Results

C701

4 Concluding remarks

C707

References

C707

\section{Introduction}

When a hot fluid and a cold fluid are separated by a conducting vertical plate heat is transferred through the plate and natural convection boundary layers form on both sides, with the boundary layer in the hot fluid flowing downwards and that in the cold fluid flowing upwards. This heat transfer system is very common in many industrial settings for example in solar collectors, nuclear reactors, electronic equipment and HVAC of buildings. A common configuration of this flow is the partitioned cavity geometry illustrated in Figure 1. In this configuration the left and right outer walls are held at fixed 'hot' and 'cold' temperatures respectively. The top and bottom walls are adiabatic and the central vertical wall is conducting. In fully developed flow the cores of the cavities are stratified and nearly quiescent. The natural convection boundary layers on the vertical walls flow onto the horizontal walls forming intrusions driving clockwise circulations around the cavities. The important governing parameters for this cavity flow are the Rayleigh number, Prandtl number and cavity aspect ratio $(A=H / W)[1]$. We refer to the coupled boundary layers on the conducting wall as 'conjugate natural convection boundary layers'.

The prior work in this configuration is predominately limited to the study of 


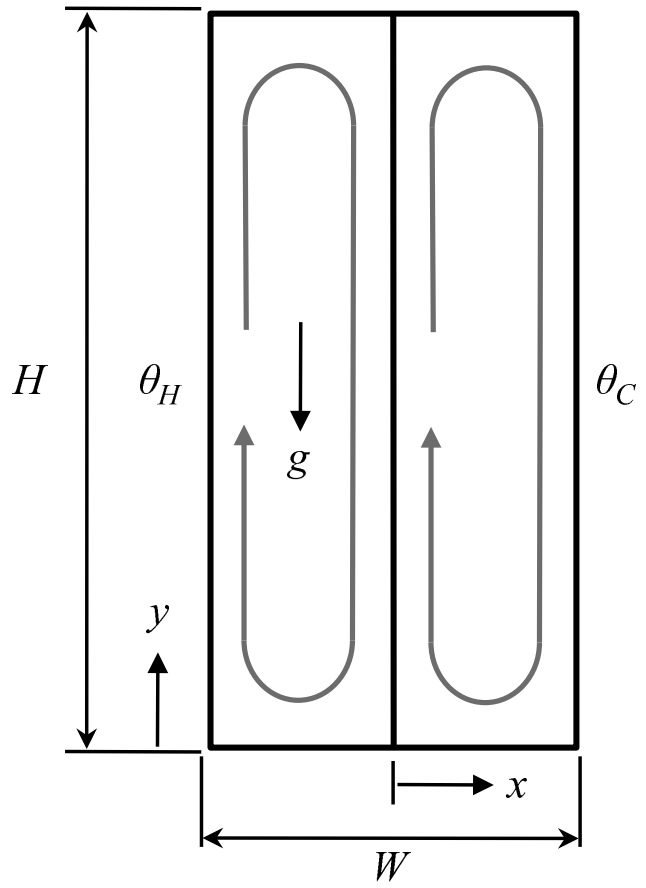

(a)

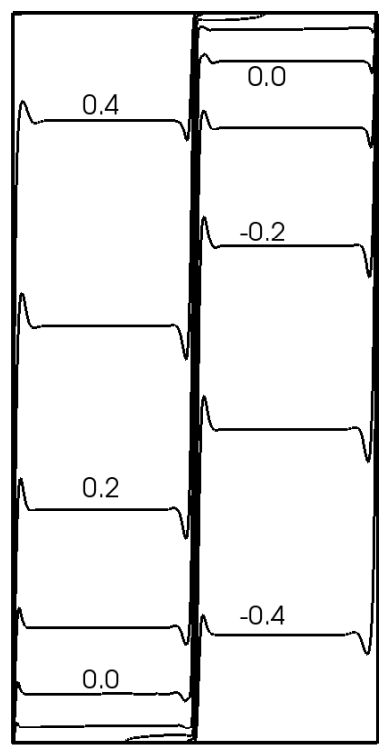

(b)

Figure 1: Configuration studied with flow circulation and coordinate system (a). Contours of mean temperature illustrated at $\mathrm{Ra}=1.4 \times 10^{10}$ and $A=2$ in (b).

the mean heat transfer behaviour $[2,3]$. The unsteady characteristics and the stability properties of the flow are also important. From an engineering design point of view, understanding the modes of oscillation may lead to opportunities for heat transfer enhancement or control. In the non-partitioned cavity configuration prior work has shown that the transition from steady flow to time dependent flow depends on Rayleigh number, Prandtl number and cavity aspect ratio and the mechanism by which this transition occurs depends on the Prandtl number $[4,5,6,1,7,8,9,10,11,12]$. In this work we consider high Prandtl number flow in which the first unstable mode of 
oscillation is associated with the natural convection boundary layers [10]. At $\operatorname{Pr}=4$ and $A=1$ the transition from steady to unsteady flow occurs at $\mathrm{Ra}=2.51 \times 10^{10}[10]$. The critical Rayleigh number for this bifurcation is not well defined for $\operatorname{Pr}=7.5$ but is thought to be greater than $10^{11}$ [13]. These critical Rayleigh numbers represent a value for the absolute instability of the system, unconditional on external forcing. At a smaller Rayleigh number natural convection boundary layers can be convectively unstable, meaning that if a perturbation of frequency $f_{c}$ is imposed on the flow, the signal will be amplified by the boundary layer. Once the exciting perturbation ceases the oscillations will decay. In isothermal cavity flow the critical Rayleigh number for convective instability in the boundary layers is $\mathrm{Ra} \approx 10^{6}$ at $\operatorname{Pr}=7.5$ [11]. Convectively unstable boundary layers can become absolutely unstable if there is a reinforcing feedback mechanism. In conjugate boundary layers, a feedback mechanism exists through the temperature field across the conducting wall. We show that this coupling allows the boundary layers on either side of the conducting wall to become absolutely unstable at a lower Rayleigh number than for a similar aspect ratio isothermal cavity. We use direct numerical simulations to locate the critical Rayleigh number for this flow configuration.

\section{Numerical formulation}

The two dimensional Navier-Stokes equations for incompressible flow with the Oberbeck-Boussinesq approximation for buoyancy are [11],

$$
\begin{aligned}
& \frac{\partial u_{i}}{\partial x_{i}}=0 \\
& \frac{\partial u_{i}}{\partial t}+\frac{\partial\left(u_{i} u_{j}\right)}{\partial x_{j}}=-\frac{\partial p}{\partial x_{i}}+\frac{\operatorname{Pr}}{R^{1 / 2}} \frac{\partial^{2} u_{i}}{\partial x_{j} \partial x_{j}}-\operatorname{Pr} \phi \\
& \frac{\partial \phi}{\partial t}+\frac{\partial\left(u_{j} \phi\right)}{\partial x_{j}}=\frac{1}{R a^{1 / 2}} \frac{\partial^{2} \phi}{\partial x_{j} \partial x_{j}},
\end{aligned}
$$


where $\operatorname{Pr}=v / \alpha$ is the Prandtl number, and the Rayleigh number is $\mathrm{Ra}=$ $\mathrm{g} \beta \Delta \theta \mathrm{H}^{3} / v \alpha$. The kinematic viscosity and thermal diffusivity of the fluid are $\nu$ and $\alpha, \beta$ is the coefficient of thermal expansion, and gravitational acceleration is $\mathrm{g}$. The dimensional velocity $\left(\tilde{\mathrm{U}}_{i}\right)$, temperature $(\tilde{\theta})$, pressure $(\tilde{\mathrm{P}})$, time $(\tilde{\mathrm{T}})$ and length $\left(\tilde{\mathrm{X}}_{i}\right)$ are made non-dimensional as $\mathrm{U}_{i}=\tilde{\mathrm{U}}_{i} / \mathrm{U}^{*}, \phi=$ $\left(\tilde{\theta}-\tilde{\theta}_{r}\right) / \Delta \tilde{\theta}, p=\tilde{P} / \rho U^{* 2}, t=\tilde{T} U^{*} / H$ and $x_{i}=\tilde{X}_{i} / H$ respectively. $\Delta \tilde{\theta}=$ $\tilde{\theta}_{H}-\tilde{\theta}_{C}$ is the temperature difference between the hot and cold walls and the reference temperature $\tilde{\theta}_{\mathrm{r}}=0.5\left(\tilde{\theta}_{\mathrm{H}}+\tilde{\theta}_{\mathrm{C}}\right)$. The characteristic velocity $\mathrm{U}^{*}=$ $\kappa \mathrm{Ra}^{1 / 2} / \mathrm{H}$ [5]. The height of the cavity is $\mathrm{H}$ and $\mathrm{\kappa}$ is the fluid conductivity.

The discretised governing equations were solved in finite volume form on a non-staggered Cartesian grid. The spatial derivatives were discretised using second order central finite differences except for the advective terms which were discretised using the third order accurate QUICK scheme [14]. The advective terms were advanced in time using the second order Adams-Bashforth scheme while the viscous terms were advanced using the Crank-Nicolson scheme [15]. The system of equations was solved using the BICGSTAB solver with a multigrid Jacobi pre-conditioner [15]. The code has been extensively benchmarked and validated in similar flows [16].

We performed a series of simulations over the range $\mathrm{Ra}=1.0-1.6 \times 10^{10}$ at a Prandtl number of $\operatorname{Pr}=7.5$ and with $A=1-2$ with an initial condition of $(\phi, \mathfrak{u}, v)=0$. These simulations were continued until the flow reached full development and the flow was statistically invariant with time. The grid size at the vertical walls was $\Delta x_{w}=5 \times 10^{-5}$ and $\Delta y=0.002$. The grid is uniform in the vertical $(y)$ direction and has $0.5-1 \%$ linear stretching in the horizontal $(x)$ direction giving $n_{y}=517$ and $n_{x}=776$ computational nodes at $A=2$ and $n_{x}=1032$ at $A=1$. A time step of $\Delta t=7.1 \times 10^{-5}$ was used. Figure 1 indicates the coordinate system used in this study, with $x$ zero at the partition wall, positive in the right cavity and negative in the left cavity. 


\section{$3 \quad$ Results}

The development of the flow from an initial condition $\phi=0$ is shown in Figure 2 for $\mathrm{Ra}=1.4 \times 10^{10}$ and $A=2$, where temperature $\phi$ is plotted with time at a location on the bottom left side of the conducting wall. A short time after start-up, natural convection boundary layers form on the vertical isothermal walls and flow into the intrusions with the remainder of the cavity quiescent. After $t \approx 3.5$, the horizontal intrusions meet the vertical partition wall and the cavity begins to fill up and stratify. Waves generated in this region propagate across the cavity and excite oscillations in the vertical boundary layer on the isothermal walls. Between $t=30-50$, the oscillations related to the start-up flow are reduced but the oscillations on the conducting wall are self-sustaining and persist. These oscillations are amplified along the natural convection boundary layers on the vertical walls but are also present at much reduced magnitude in the intrusions and cavity cores. From $t=50-400$ the mean flow field asymptotically approaches its final solution. The initially multi-modal oscillations in the flow field become increasingly dominated by a single mode of oscillation shown in Figure 2(inset).

All the simulations in this study are well above $\mathrm{Ra}_{\mathrm{c}}=10^{6}$, so the natural convection boundary layers on both the isothermal and conducting walls are expected to be convectively unstable and amplify oscillations within a specific band of frequencies $f_{1}$ to $f_{2}$, where $f_{1}$ and $f_{2}$ may vary with height or local Rayleigh number. Figure 2 demonstrates that the flow in the partitioned cavity with a conjugate boundary layer is not only convectively unstable but is absolutely unstable at $\operatorname{Pr}=7.5, A=2$ and $\mathrm{Ra}=1.4 \times 10^{10}$ as the oscillations are sustained with no external forcing. The critical Rayleigh number for a non-partitioned isothermal cavity is not known for $\operatorname{Pr}=7.5$ flow but we performed additional numerical simulations of an isothermal cavity up to $\mathrm{Ra}=2 \times 10^{10}$ at $A=4$ and $\operatorname{Pr}=7.5$ and confirm that the flow does not bifurcate at these values. The instability observed here in the partitioned cavity flow therefore occurs at a lower Rayleigh number than in isothermal cavity flow and has a different character, being predominately single modal 


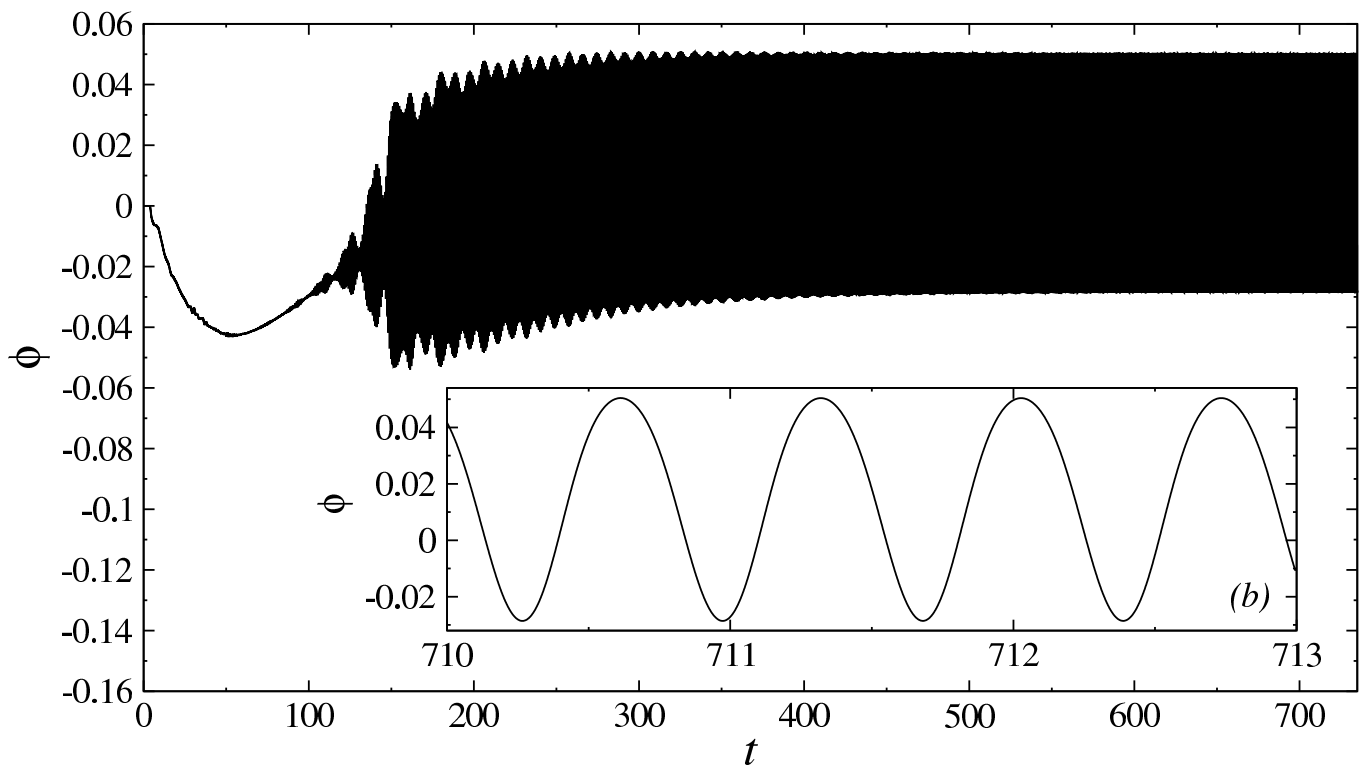

Figure 2: Time trace of temperature $\phi$ for $\mathrm{Ra}=1.4 \times 10^{10}$ and $A=2$ at $y=0.1$ and $x=-0.005$. The inset plot illustrates the dominant mode of oscillation at full development.

rather than weakly turbulent.

The temperature perturbation is defined as $\phi^{\prime}=\phi-\langle\phi\rangle$ where \langle\rangle indicates time averaging. Figure 3 plots $\phi^{\prime}$ along the conducting wall (y-direction) at time $t=t_{1}$ and a short time later $t=t_{1}+0.071$, on either side of the conducting wall. The direction of the travelling wave is inferred on either side of the conducting wall. On the left side of the wall the boundary layer is travelling down the wall in the negative $y$ direction. On this same side below $y \approx 0.7$, the wave travels in the direction of boundary layer flow and is strongly amplified in this direction as illustrated by the arrows. Above $y \approx 0.7$ the wave travels against the direction of flow and is amplified in the positive $y$ direction. This perturbation is not generated in the flow but is apparently conducted through the wall from the conjugate boundary layer. 
The phase lag between the oscillations on either side of the wall is a function of the horizontal distance. The transmission of the oscillations away from the partition wall is inferred from the temperature variance $\left\langle\phi^{\prime} \phi^{\prime}\right\rangle^{0.5}$ in Figure 4.

This coupling mechanism between the two boundary layers provides a feedback mechanism which allows the system to become absolutely unstable at sufficiently high Rayleigh number. This instability is illustrated as follows. If an initial forcing is applied to the system at an unstable frequency $f$, the signal will be amplified along one boundary layer, conducted through the wall and then be amplified in the opposing direction on the other side of the partition wall. If the flow is above a critical Rayleigh number $R a_{p}$, then the perturbations grow in time until ultimately the growth of the oscillations in the system is limited by dissipation in the boundary layer. In this way the instability appears to be a convective boundary layer instability which is able to persist through a feedback loop.

Additional simulations were performed to determine the range of Rayleigh numbers for which the system is unstable at $A=2$ and $A=1$. At $A=2$ results are presented here for $\mathrm{Ra}=1.0 \times 10^{10}$ where the flow is initiated from the $\mathrm{Ra}=1.4 \times 10^{10}$ flow field. The sudden change in Rayleigh number introduces a large perturbation into the flow. Figure 5 gives the time trace of temperature $\phi$. The flow rapidly adjusts to the new Rayleigh number and large oscillations appear in the vertical boundary layers. The oscillations grow spatially in the direction of mean flow along the boundary layers indicating that the flow is convectively unstable. Temporally though, the oscillations decay with time asymptotically approaching a steady solution, indicating that the flow is not absolutely unstable. At $\mathrm{Ra}=1.2 \times 10^{10}$ and $\mathrm{A}=2$ a simulation was performed initiated from a quiescent initial condition and the flow was found to be absolutely unstable, indicating the transition from convectively unstable flow to absolutely unstable found occurs between $\mathrm{Ra}=1.0 \times 10^{10}$ and $\mathrm{Ra}=1.2 \times 10^{10}$ at $\mathrm{A}=2$.

A smaller aspect ratio simulation at $A=1$ was performed at $\mathrm{Ra}=1.4 \times 10^{10}$ from the quiescent $\phi=0$ initial condition and the flow was found to be 


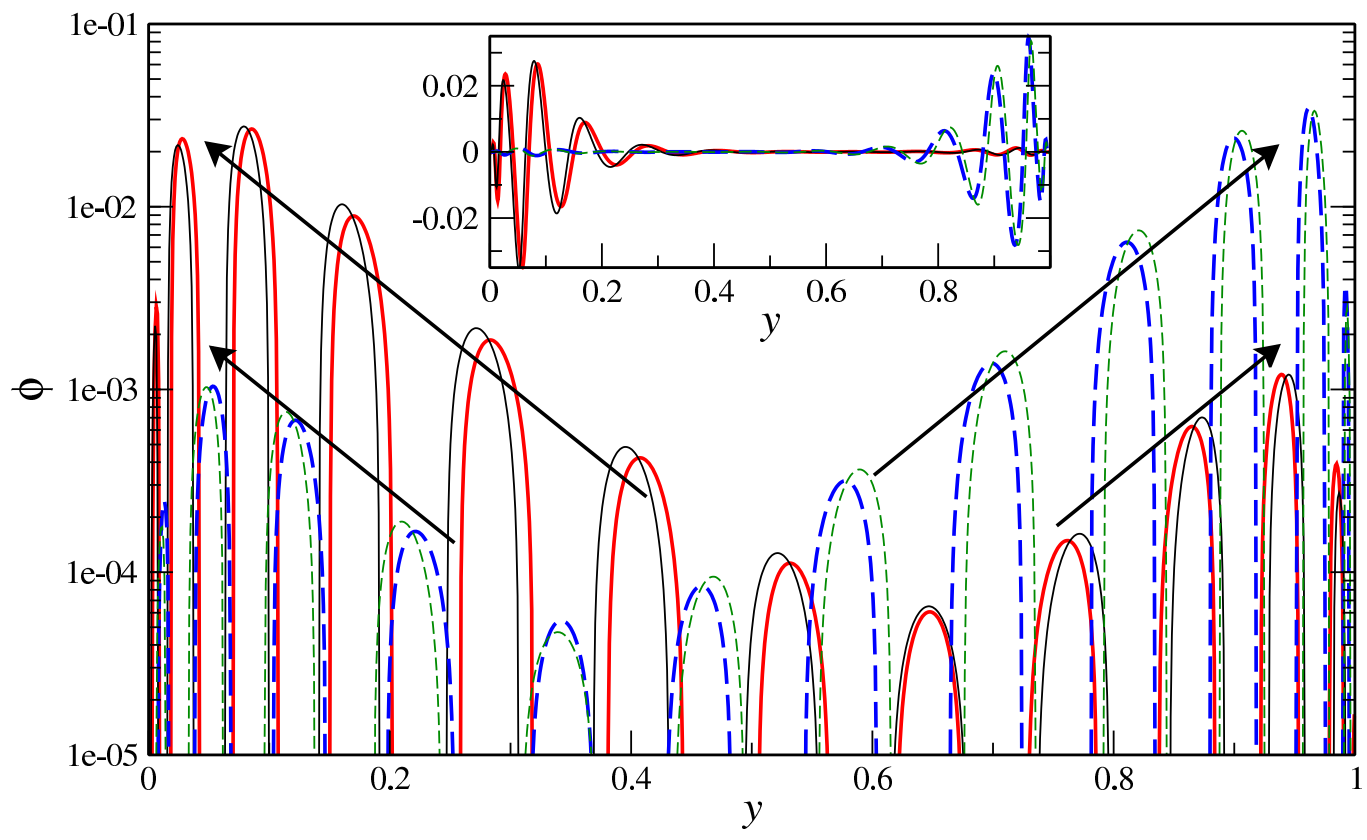

Figure 3: $\phi^{\prime}$ at $R a=1.4 \times 10^{10}$ and $A=2$ plotted against vertical location at two horizontal locations, $x=-0.002$ (solid lines) just to the left of the conducting wall and $x=0.002$ (dashed lines) just to the right of the wall. Thick lines indicate data at $t=t_{1}$ and thin lines data at $t_{2}=t_{1}+0.071$. Arrows indicate direction of the waves in the coupled boundary layers. Inset is the same data presented on a linear scale.

absolutely unstable. A further test was performed at $\mathrm{Ra}=1.2 \times 10^{10}$ at $A=1$ and found to be convectively unstable but not absolutely unstable, as opposed to flow at $A=2$ at the same Rayleigh number, indicating that the critical Rayleigh number has an aspect ratio dependence. This difference in stability properties is attributed to the small changes in the mean flow field between the $A=1$ and $A=2$ results. Another possibility is that the transmission of waves around the cavity is altered and that this makes the flow more stable. 


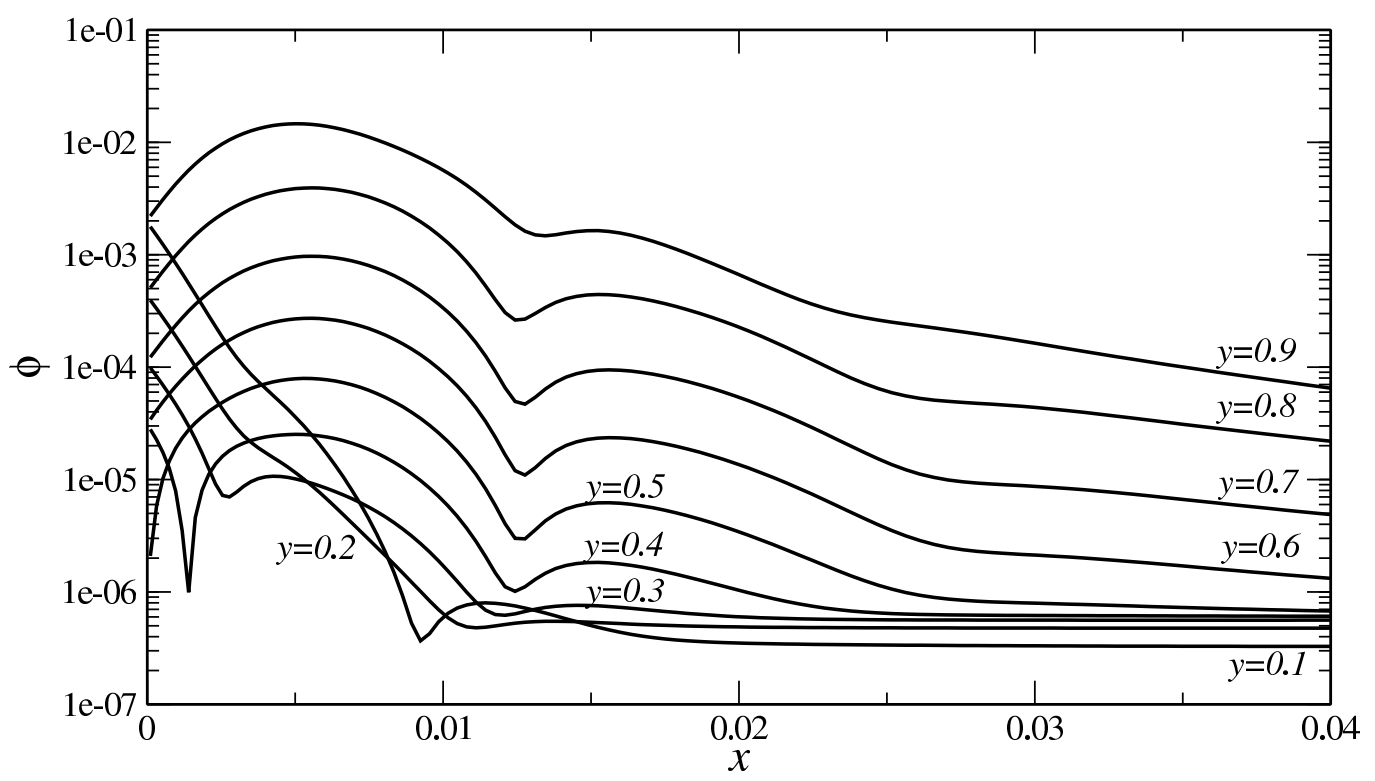

Figure 4: $\left\langle\phi^{\prime} \phi^{\prime}\right\rangle^{1 / 2}$ at $\mathrm{Ra}=1.4 \times 10^{10}$ and $A=2$ with distance from the conducting wall in the right cavity.

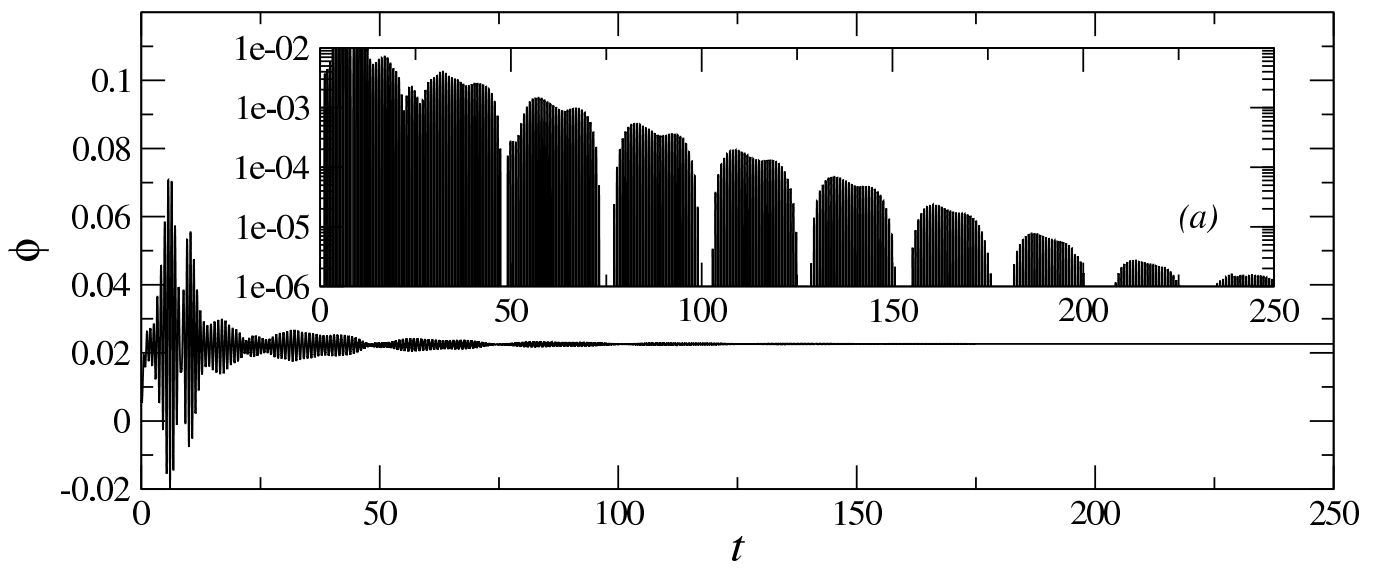

Figure 5: Time trace of temperature $\phi$ for $\mathrm{Ra}=1.0 \times 10^{10}$ and $\mathrm{A}=2$ at $y=0.125$ and $x=-0.005$. The inset gives $\phi^{\prime}=\phi-\langle\phi\rangle$ on a log scale where $\langle\phi\rangle$ is the final mean non-dimensional temperature at this location. 


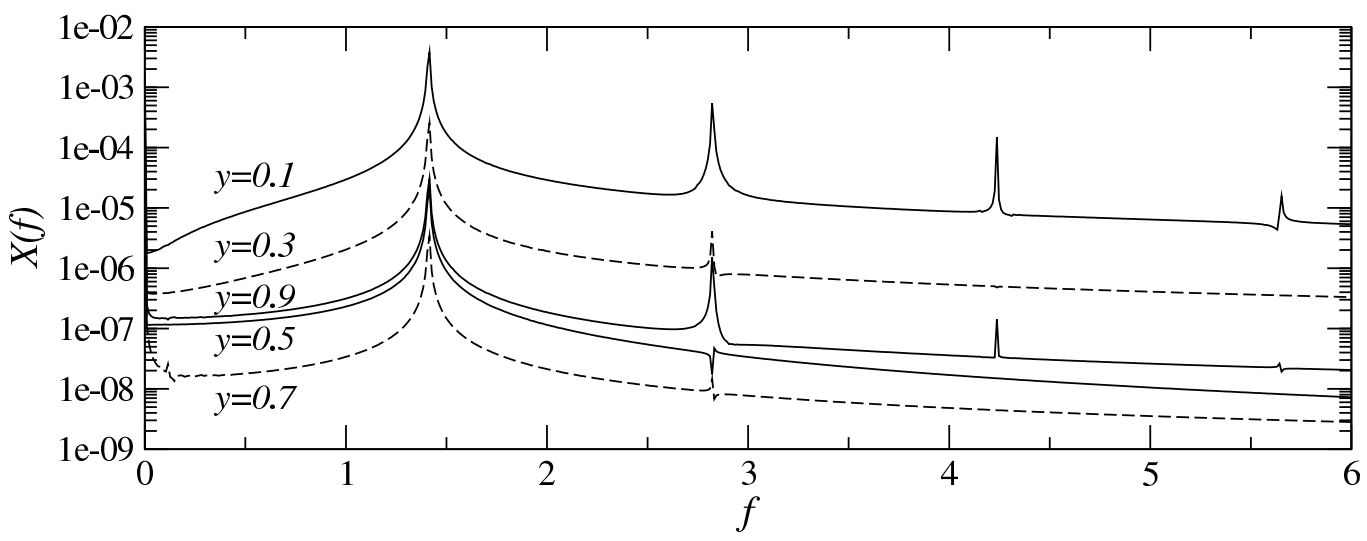

FiguRE 6: The discrete Fourier transform of velocity $u(t)$ from $t=650-700$ at $y=0.1-0.9$ and $x=-0.005$ (at the conducting wall) with $\mathrm{Ra}=1.4 \times 10^{10}$ and $A=2$.

Figure 6 shows the spectrum of the velocity $u$ signal at locations along the left side of the conducting wall for the $\mathrm{Ra}=1.4 \times 10^{10}$ and $A=2$ result. The dominant mode of oscillation has a frequency of $f=1.413$ with higher harmonics of this signal also present. This dominate frequency is constant across the height of the cavity indicating that the oscillations induced in the upstream end of the boundary layer, which travel against the mean flow, have the same frequency as the oscillations in the downstream end of the boundary layer travelling in the opposite direction. Normalising this dominant mode with the natural convection boundary layer time scale gives $f^{+}=f \gamma^{1 / 3} /(g \beta \Delta \theta)^{2 / 3} \approx 0.0145$, slightly smaller than the most unstable mode in convectively unstable isothermal cavity flow [17]. The normalised frequencies of this oscillation in all the simulations performed in this study range over $\mathrm{f}^{+}=0.0142-0.0147$ irrespective of aspect ratio or Rayleigh number. 


\section{Concluding remarks}

Flow in a partitioned cavity with isothermal outer walls and a conducting partition wall is shown to becomes oscillatory at $\operatorname{Pr}=7.5$ between $\mathrm{Ra}_{\mathrm{p}}=$ $1.0-1.2 \times 10^{10}$ for $A=2$, and between $R a_{p}=1.2-1.4 \times 10^{10}$ for $A=1$. The bifurcation is manifested as a single mode of oscillation with $\mathrm{f}^{+} \approx 0.0145$ originating in the conjugate natural convection boundary layers formed on either side of a conducting wall. The instability appears to be a result of a convective boundary layer instability which becomes absolutely unstable via the thermal coupling between the boundary layers on either side of the partition wall. The results show that, unlike isothermal cavity flow, partitioned cavity flow with conjugate natural convection boundary layers has an additional regime of behaviour between the convectively unstable regime and weakly turbulent flow regime.

Acknowledgements We acknowledge the support of the Australian Research Council.

\section{References}

[1] S. Paolucci and D. R. Chenoweth. Transition to chaos in a differentially heated vertical cavity. J. of Fluid Mech., 201:379-410, 1989. doi:10.1017/S0022112089000984 C697, C698

[2] R. Anderson and A. Bejan. Heat transfer through single and double vertical walls in natural convection: Theory and experiment. Int. J. Heat Mass Transfer, 24(10):1611-1620, 1981. doi:10.1016/0017-9310(81)90069-7 C698

[3] Tatsuo Nishimura, Mitsuhiro Shiraishi, Fumio Nagasawa, and Yuji Kawamura. Natural convection heat transfer in enclosures with multiple 
vertical partitions. Int. J. Heat Mass Transfer, 31(8):1679-1686, 1988. doi:10.1016/0017-9310(88)90280-3 C698

[4] J. W. Elder. Turbulent free convection in a vertical slot. J. of Fluid Mech., 23:99-111, 1965. doi:10.1017/S0022112065001258 C698

[5] J. Patterson and J. Imberger. Unsteady natural convection in a rectangular cavity. J. of Fluid Mech., 100:65-86, 1980. doi:10.1017/S0022112080001012 C698, C700

[6] G. N. Ivey. Experiments on transient natural convection in a cavity. J. of Fluid Mech., 144:389-401, 1984. doi:10.1017/S0022112084001658 C698

[7] J. C. Patterson and S. W. Armfield. Transient features of natural convection in a cavity. J. Fluid Mech., 219:469-497, 1990. doi:10.1017/S0022112090003032 C698

[8] P. Le Quéré. Transition to unsteady natural convection in a tall water-filled cavity. Phys. Fluids A, 2(4):503-515, 1990. doi:10.1063/1.857750 C698

[9] S. W. Armfield and John C. Patterson. Wave properties of natural-convection boundary layers. J. Fluid Mech., 239:195-211, 1992. doi:10.1017/S0022112092004373 C698

[10] R. J. A. Janssen and R. A. W. M. Henkes. Influence of Prandtl number on instability mechanisms and transition in a differentially heated square cavity. J. of Fluid Mech., 290:319-344, 1995. doi:10.1017/S0022112095002539 C698, C699

[11] S. Armfield and R. Janssen. A direct boundary-layer stability analysis of steady-state cavity convection flow. Int. J. Heat Fluid Flow, 17(6):539-546, 1996. doi:10.1016/S0142-727X(96)00065-3 C698, C699 
[12] J. C. Patterson, T. Graham, W. Schop̈f, and S. W. Armfield. Boundary layer development on a semi-infinite suddenly heated vertical plate. $J$. Fluid Mech., 453:39-55, 2002. doi:10.1017/S0022112001006553 C698

[13] R. A. W. M. Henkes. Turbulent natural convection boundary layers. PhD thesis, University of Delft, 1990. C699

[14] B. P. Leonard. A stable and accurate convective modelling procedure based on quadatic upstream interpolation. Comp. Meth. Appl. Eng., 19:59-98, 1979. doi:10.1016/0045-7825(79)90034-3 C700

[15] J. H. Ferziger and M. Perić. Computational Methods for Fluid Dynamics. Springer, 2002. C700

[16] S. E. Norris. A Parallel Navier-Stokes Solver for Natural Convection and Free Surface Flow. PhD thesis, University of Sydney, 2000. C700

[17] R. Janssen and S. Armfield. Stability properties of the vertical boundary layers in differentially heated cavities. Int. J. Heat Fluid Flow, 17(6):547-556, 1996. doi:10.1016/S0142-727X(96)00077-X C706

\section{Author addresses}

1. N. Williamson, School of Aerospace, Mechanical and Mechatronic Engineering, University of Sydney, Australia. mailto:nicholas.williamson@sydney .edu.au

2. S. W. Armfield, School of Aerospace, Mechanical and Mechatronic Engineering, University of Sydney, Australia. 